\title{
New construction of error-tolerant pooling designs
}

\author{
R. Ahlswede and H. Aydinian \\ Department of Mathematics \\ University of Bielefeld \\ POB 100131, D-33501 Bielefeld, Germany \\ Email: ahlswede@math.uni-bielefeld.de \\ ayd@math.uni-bielefeld.de
}

\begin{abstract}
We present a new class of error-tolerant pooling designs by constructing $d^{z}$-disjunct matrices associated with subspaces of a finite vector space.
\end{abstract}

Keywords Group testing, Nonadaptive algorithm, Pooling designs, $d^{z}$-disjunct matrix

\section{Introduction}

Combinatorial group testing has various practical applications [8], [9]. In the classical group testing model we have a set $[n]=\{1, \ldots, n\}$ of $n$ items containing at most $d$ defective items. The basic problem of group testing is to identify the set of all defective items with a small number of group tests. Each group test, also called a pool, is a subset of items. It is assumed that there is a testing mechanism that for each subset $A \subset[n]$ gives one of two possible outcomes: negative or positive. The outcome is positive if $A$ contains at least one defective and is negative otherwise.

A group testing algorithm is called nonadaptive if all tests are specified without knowledge of the outcomes of other tests. Traditionally, a nonadaptive group testing algorithm is called a pooling design. Pooling designs have many applications in molecular biology, such as DNA screening, nonunique probe selection, gene detection, etc. (see [9], [10]).

A pooling design is associated with a $(0,1)$ - inclusion matrix $M=\left\{m_{i j}\right\}$, where the rows are indexed by tests $A_{1}, \ldots, A_{t} \subset[n]$, the columns are indexed by items $1, \ldots, n$, and $m_{i j}=1$ if and only if $j \in A_{i}$. The major tool used for construction of pooling designs are $d$-disjunct matrices. Let $M$ be a binary $t \times n$ matrix where the columns $C_{1}, \ldots, C_{n}$ are viewed as subsets of $[t]=\{1, \ldots, t\}$ represented by their characteristic vectors. Then $M$ is called $d-$ disjunct if no column is contained in the union of $d$ others. The notion of $d$-disjunctness was introduced by Kautz and Singleton [14]. They proved that a $d$-disjunct matrix $M$ can 
identify up to $d$ defective items. $d$-disjunct matrices are also known as $d$-cover free families studied in extremal set theory [7].

The maximal $d$ for which $M$ is $d$-disjunct is called the degree of disjunctness and is denoted by $d_{\max }$. Note that $d$-disjunctness of a pooling design is a sufficient, but not a necessary condition for identification of $d$ defectives. However a $d$-disjunct pooling design has an advantage of a very simple decoding. Removing from the set of items all items in negative pools we get all defectives (see [9] for details).

A pooling design is called error-tolerant if it can detect/correct some errors in test outcomes. Biological experiments are known to be unreliable (see [9]), which, in fact, is a practical motivation for constructing efficient error-tolerant pooling designs.

For error correction in tests the notion of a $d^{z}$-disjunct matrix was introduced in [17]. A $d$-disjunct matrix is called $d^{z}$-disjunct if for any $d+1$ of its columns $C_{i_{1}}, \ldots, C_{i_{d+1}}$ we have $\left|C_{i_{1}} \backslash\left(C_{i_{2}} \cup \ldots \cup C_{i_{d+1}}\right)\right| \geq z$. In fact, the $d^{1}$-disjunctness is simply the $d$-disjunctness. A $d^{z}$-disjunct matrix can detect $z-1$ errors and correct $\left\lfloor\frac{z-1}{2}\right\rfloor$ errors (see e.g. [10] or [9]). Constructions of $d^{z}$-disjunct matrices are given by many authors (see [2], [17], [18], [10]).

Most known constructions of $d^{z}$-disjunct matrices are matrices with a constant column weight. Let $M$ be a binary $t \times n$ matrix with a constant column weight $k$ and let $s$ be the maximum size of intersection (number of common ones) between two different columns. Kautz and Singleton [14] observed that then $M$ is $d$-disjunct with $d=\left\lfloor\frac{k-1}{s}\right\rfloor$. Moreover, for integers $0 \leq s<k<t$ the maximum number $n(d, t, w)$ for which there exists such a disjunct matrix is upper bounded by

$$
n(d, t, k) \leq\left(\begin{array}{c}
t \\
s+1
\end{array}\right) /\left(\begin{array}{c}
k \\
s+1
\end{array}\right)
$$

Note that the columns of $M$ considered as the family $\mathcal{F}$ of $k$-subsets of $[t]$ (called blocks) form an $(s+1, k, t)$-packing, that is each $(s+1)$-subset of $[t]$ is contained in at most one block of $\mathcal{F}$. Note also that equality in $(1.1)$ is attained if and only if $\mathcal{F}$ is an $(s+1, k, t)$-Steiner system (each $(s+1)$-subset is contained in precisely one block).

Thus, packing designs can be used for construction of $d$-disjunct matrices. However, construction of good $(s+1, k, t)$-packings, in general, is known to be a difficult combinatorial problem. Several other constructions (see [9, Ch.3]) of disjunct matrices are also based on combinatorial structures or error correcting codes. We note that $(s+1, k, t)$-packings can also be described in terms of codes in the Johnson graph $J(n, k)$ (or Johnson scheme) with minimum distance $d_{J}=k-s$. It seems natural to try other distance regular graphs (see [4] for definitions), for construction of $d$-disjunct matrices, using the idea of packings.

In this paper we construct new error-tolerant pooling designs associated with finite vector spaces. In Section 2 we briefly review some known constructions of disjunct matrices based on partial orders and determine the degree of disjunctness for the construction proposed by Ngo and Du [18]. Our main results are stated and proved in Section 3. We present a construction of $d^{z}$-disjunct matrices based on packings in finite projective spaces. For certain parameters the construction gives better performance than previously known ones. 


\section{$2 \quad d^{z}$-disjunct matrices from partial orders}

Macula [16] proposed a simple direct construction of $d$-disjunct matrices. Given integers $1 \leq d<k<m$, let $M=\left(m_{i j}\right)$ be an $\left(\begin{array}{c}m \\ d\end{array}\right) \times\left(\begin{array}{c}m \\ k\end{array}\right)$ matrix where the rows are indexed by elements of $\left(\begin{array}{c}{[m]} \\ d\end{array}\right)$, the columns are indexed by the elements of $\left(\begin{array}{c}{[m]} \\ k\end{array}\right)$, and $m_{i j}=1$ if we have containment relation between the subsets corresponding to the $i$ th row and the $j$ th column, otherwise $m_{i j}=0$. Note that each column has weight $\left(\begin{array}{l}k \\ d\end{array}\right)$ and each row has weight $\left(\begin{array}{c}m-d \\ k-d\end{array}\right)$. Macula showed that $M$ is a $d$-disjunct matrix and $d_{\max }=d$.

Similar constructions, using different posets, were given by several authors. Ngo and Du [18] extended Macula's construction to some geometric structures. In particular they considered the following construction of a $d$-disjunct matrix $M_{q}(m, d, k)$ associated with finite vector spaces. Let $G F(q)^{m}$ be the $m$-dimensional vector space over $G F(q)$. The set of all subspaces of $G F(q)^{m}$, called projective space, is denoted by $\mathcal{P}_{q}(m)$. Recall that $\mathcal{P}_{q}(m)$ ordered by containment is known as the poset of linear spaces (or linear lattice). Given an integer $0 \leq k \leq m$, the set of all $k$-dimensional subspaces ( $k$-spaces for short) of $G F(q)^{m}$ is called a Grassmannian and denoted by $\mathcal{G}_{q}(m, k)$. Thus, we have $\bigcup_{0 \leq k \leq m} \mathcal{G}_{q}(m, k)=\mathcal{P}_{q}(m)$. A graph associated with $\mathcal{G}_{q}(m, k)$ is called the Grassmann graph, when two vertices (elements of $\left.\mathcal{G}_{q}(m, k)\right) V$ and $U$ are adjacent iff $\operatorname{dim}(V \cap U)=k-1$ (see [4] for more insight). It is known that the size of the Grassmannian $\left|\mathcal{G}_{q}(m, k)\right|$ is determined by the $q$-ary Gaussian coefficient $\left.\left[\begin{array}{c}m \\ k\end{array}\right]_{q} ; k=0,1, \ldots, m\left(\left[\begin{array}{c}m \\ 0\end{array}\right]_{q} \triangleq 1\right)\right)$,

$$
\left|\mathcal{G}_{q}(m, k)\right|=\left[\begin{array}{c}
m \\
k
\end{array}\right]_{q}=\frac{\left(q^{m}-1\right)\left(q^{m-1}-1\right) \cdots\left(q^{m-k+1}-1\right)}{\left(q^{k}-1\right)\left(q^{k-1}-1\right) \cdots(q-1)} .
$$

For integers $1 \leq r<k<m$, the $\left[\begin{array}{c}m \\ r\end{array}\right]_{q} \times\left[\begin{array}{c}m \\ k\end{array}\right]_{q}$ incidence matrix $M_{q}(m, r, k)=\left(m_{i j}\right)$ is defined as follows. The rows and the columns are indexed by the elements of $\mathcal{G}_{q}(m, r)$ and $\mathcal{G}_{q}(m, k)$ (given in a fixed ordering), respectively, and $m_{i j}=1$ if we have containment relation, otherwise $m_{i j}=0$. Note that each column of $M_{q}(m, r, k)$ has weight $\left[\begin{array}{l}k \\ r\end{array}\right]_{q}$ and each row has weight $\left[\begin{array}{c}m-r \\ k-r\end{array}\right]_{q}$. Ngo and Du showed that $M_{q}(m, r, k)$ is an $r$-disjunct matrix. However D'yachkov et al. [10] observed that the degree of disjunctness of $M_{q}(m, r, k)$ can be much bigger than $r$. Moreover, the construction can in general tolerate many errors.

Theorem DHMVW [10]

For $k-r \geq 2$ and $d<\frac{q\left(q^{k-1}-1\right)}{q^{k-r}-1}$, the matrix $M_{q}(m, r, k)$ is $d^{z}$-disjunct with

$$
z \geq\left[\begin{array}{l}
k \\
r
\end{array}\right]_{q}-d\left[\begin{array}{c}
k-1 \\
r
\end{array}\right]_{q}+(d-1)\left[\begin{array}{c}
k-2 \\
r
\end{array}\right]_{q} .
$$

The bound is tight for $d \leq q+1$.

Note that the maximum number $d$ in (2.2) for which $z>0$ is $d=\frac{q\left(q^{k-1}-1\right)}{q^{k-r}-1}$. Thus, the theorem tells us that $d_{\max } \geq \frac{q\left(q^{k-1}-1\right)}{q^{k-r}-1}$. In fact, we determine $d_{\max }$ for every $M_{q}(m, r, k)$.

Theorem 1 For integers $1 \leq r<k<m$, the degree of disjunctness of $M_{q}(m, r, k)$ equals

$$
d_{\max }=\frac{q\left(q^{r}-1\right)}{q-1} .
$$


Proof. Let $V \in \mathcal{G}_{q}(m, k)$. We wish to determine the minimum size of a set of $k$-spaces which cover (contain) all $r$-spaces of $V$. Suppose $U_{1}, \ldots, U_{p} \in \mathcal{G}_{q}(m, k)$ is a minimal covering of the $r$-spaces of $V$. Without loss of generality, we may assume that $\operatorname{dim}\left(U_{i} \cap V\right)=k-1$ for $i=1, \ldots, p$. Therefore, $W_{1}=U_{1} \cap V, \ldots, W_{p}=U_{p} \cap V$ can be viewed as a set of hyperplanes of $\mathcal{P}_{q}(k)$ that cover all $r$-spaces of $\mathcal{P}_{q}(k)$. Let now $A_{i} \in \mathcal{P}_{q}(k)$ be the orthogonal space of $W_{i}$; $i=1, \ldots, p$. Thus, $\mathcal{A}=\left\{A_{1}, \ldots, A_{p}\right\}$ is a set of one dimensional subspaces, that is points, in $\mathcal{P}_{q}(k)$. By the principle of duality, every $(k-r)$-space of $\mathcal{P}_{q}(k)$ contains an element of $\mathcal{A}$. To complete the proof we use the following result.

Theorem BB [3] Let $\mathcal{A} \subset G F(q)^{m} \backslash\{0\}$ have a non-empty intersection with every $(k-r)$ space of $\mathcal{P}_{q}(k)$. Then $|\mathcal{A}| \geq\left(q^{r+1}-1\right) /(q-1)$, with equality if and only if $\mathcal{A}$ consists of $\left(q^{r+1}-1\right) /(q-1)$ points of an $(r+1)$-space of $\mathcal{P}_{q}(k)$.

It is clear now that $d_{\max }=\left(q^{r+1}-1\right) /(q-1)-1$.

\section{New construction}

Our construction of a disjunct matrix $M$ is based on packings in $\mathcal{P}_{q}(m)$. For integers $0 \leq$ $s<k<m$, a subset $\mathcal{C} \subset \mathcal{G}(m, k)$ (with the elements called blocks) is called an $[s+1, k, m]_{q^{-}}$ packing if each $(s+1)$-space of $\mathcal{P}_{q}(m)$ is contained in at most one block of $\mathcal{C}$. This clearly means that $\operatorname{dim}(V \cap U) \leq s$ for every distinct pair $V, U \in \mathcal{C}$. $\mathcal{C}$ is called an $[s+1, k, m]_{q^{-}}$ Steiner structure if each $(s+1)$-space of $\mathcal{P}_{q}(m)$ is contained in precisely one block of $\mathcal{C}$. Let $N(m, k, s)$ denote the maximum size of an $[s+1, k, m]_{q}$-packing.

An equivalent definition of an $[s+1, k, m]_{q}$-packing can be given in terms of the subspace distance $d_{S}(V, U)$ defined (in general for any $\left.V, U \in \mathcal{P}_{q}(m)\right)$ by $d_{S}(V, U)=\operatorname{dim} V+\operatorname{dim} U-$ $2 \operatorname{dim}(V \cap U)([1],[15])$. Then clearly $d_{S}(V, U) \geq 2(k-s)$ for every pair of elements $V, U \in \mathcal{C}$. The following simple observation is an analogue of (1.1) for projective spaces. Let $M$ be the incidence matrix of an $[s+1, k, m]_{q}$-packing $\mathcal{C}$ with $s \geq 1$, that is the $t \times n$ matrix where the rows (resp. columns) are indexed by the nonzero elements of $G F(q)^{n}$ (resp. by the blocks of $\mathcal{C}$ ) given in a fixed ordering.

Lemma 1 (i) For $d \leq q^{k-s}$, the matrix $M$ is $d^{z}$-disjunct with $z=q^{k}-1-d\left(q^{s}-1\right)$. (ii) The number of columns

$$
n \leq N(m, k, s) \leq\left[\begin{array}{c}
m \\
s+1
\end{array}\right]_{q} /\left[\begin{array}{c}
k \\
s+1
\end{array}\right]_{q}
$$

with both equalities if and only if $\mathcal{C}$ is an $[s+1, k, m]_{q}$-Steiner structure.

Proof. (i) By the definition of an $[s+1, k, m]_{q}$-packing, each $(s+1)$-space is contained in at most one $k$-space of $\mathcal{C}$. Therefore, any two columns in $M$ have at most $q^{s}-1$ common ones. Hence, a column in $M$ can be covered by at most $\left\lceil\frac{q^{k}-1}{q^{s}-1}\right\rceil>q^{k-s}$ other columns. Note that in case $s \mid k$, the space $G F(q)^{k}$ can be partitioned by $s$-spaces (see [5]) and $d_{\max }=\frac{q^{k}-1}{q^{s}-1}-1$. (ii) Since the number of $(s+1)$-spaces contained in a $k$-space is $\left[\begin{array}{c}k \\ s+1\end{array}\right]_{q}$, we have the following 
packing bound $N(m, k, s) \leq\left[\begin{array}{c}m \\ s+1\end{array}\right]_{q} /\left[\begin{array}{c}k \\ s+1\end{array}\right]_{q}$ (see [1], [20], [15]). The equality in (3.1) is attained iff we have a partition of all $(s+1)$-spaces by the blocks of $\mathcal{C}$.

A challenging problem is to find Steiner structures in $\mathcal{P}_{q}(n)$. Note that no nontrivial Steiner structures, except for the case $s=0$ when we have a partition of $G F(q)^{m}$ by $k^{-}$spaces, are known. Properties of Steiner structures in $\mathcal{P}_{q}(n)$, introduced in [1] are studied in [19].

Theorem WXS [20] (KK [15]) Given integers $1<k<m$, there exists an explicit construction of an $[s+1, k, m]_{q}$-packing $\mathcal{C}$ with

$$
|\mathcal{C}|= \begin{cases}q^{(s+1)(m-k)} & \text { if } m \geq 2 k, 0 \leq s<k \\ q^{k(s+1)} & \text { if } m<2 k, 0 \leq s<m-k .\end{cases}
$$

The construction of such packings is based on Gabidulin codes [13] The explicit description (in terms of subspace codes) is given in [20] and in [15]. For completeness we describe this construction here (in terms of $[s+1, k, m]_{q}$-packings). Let $\mathbb{F}_{q}^{k \times r}$ denote the set of all $k \times t$ matrices over $G F(q)$. For $X, Y \in \mathbb{F}_{q}^{k \times r}$ the rank distance between $X$ and $Y$ is defined as $d_{R}(X, Y)=\operatorname{rank}(X-Y)$. It is known that the rank-distance is a metric [13]. Codes in metric space $\left(\mathbb{F}_{q}^{k \times r}, d_{R}\right)$ are called rank-metric codes. It is known [13] that for a rankmetric code $\mathcal{C} \subseteq \mathbb{F}_{q}^{k \times r}$ with minimum distance $d_{R}(\mathcal{C})$ one has the Singleton bound $\log _{q}|\mathcal{C}| \leq$ $\min \left\{k\left(r-d_{R}(\mathcal{C})+1\right), r\left(k-d_{R}(\mathcal{C})+1\right)\right\}$. Codes attaining this bound are called maximumrank-distance codes (MRD). An important class of rank-metric codes are Gabidulin codes [13]. They are linear MRD codes, which exist for all parameters $k, r$ and $d_{R} \leq \min \{k, r\}$. The construction of an $[s+1, k, m]_{q}$-packing from an MRD code is as follows. Consider the space $\mathbb{F}_{q}^{k \times(m-k)}(m \geq k)$. Let first $m \geq 2 k$. Then for any integer $0 \leq s \leq k$ there exists a Gabidulin code $\mathcal{C}_{G} \subset \mathbb{F}_{q}^{k \times(m-k)}$ of minimum distance $d_{R}=k-s$ and size $q^{(s+1)(m-k)}$. To each matrix $A \in \mathcal{C}_{G}$ we put into correspondence the matrix $\left[I_{k} \mid A\right] \in \mathbb{F}_{q}^{k \times m}\left(I_{k}\right.$ is the $k \times k$ identity matrix). We define now the set of $k$-spaces $\mathcal{C}(m, k, s)_{q}=\left\{\operatorname{rowspace}\left(\left[I_{k} \mid A\right]\right): A \in \mathcal{C}_{G}\right\}$. It can easily be observed now that $\operatorname{dim}(V \cap U) \leq s$ for all pairs $V, U \in \mathcal{C}(m, k, s)_{q}$. This means that $\mathcal{C}(m, k, s)_{q}$ is an $[s+1, k, m]_{q}$-packing with $\left|\mathcal{C}(m, k, s)_{q}\right|=\left|\mathcal{C}_{G}\right|=q^{(s+1)(m-k)}$. Similarly is described the $[s+1, k, m]_{q}$-packing $\mathcal{C}(m, k, s)_{q}$ for $m<2 k$. Note that for our purposes the case $m \geq 2 k$ is more important.

The following is a useful estimate for the Gaussian coefficients. A proof can be found in [6] (and in [15] for the case $q=2$ ).

Lemma 2 For integers $1 \leq k<m$ we have

$$
q^{(m-k) k}<\left[\begin{array}{c}
m \\
k
\end{array}\right]_{q}<\alpha(q) \cdot q^{(m-k) k},
$$

where $\alpha(2)=4$ and $\alpha(q)=\frac{q}{q-2}$ for $q \geq 3$.

Note that Lemma 2 in conjunction with Theorem WXS applied to our upper bound (3.1) shows that $\mathcal{C}(m, k, s)_{q}$ is nearly optimal:

$$
\left|\mathcal{C}(m, k, s)_{q}\right| \leq\left|N(n, k, s)_{q}<\alpha(q) \cdot q^{(s+1)(m-k)}=\alpha(q) \cdot\right| \mathcal{C}(m, k, s)_{q} \mid
$$


Here actually $\lim \alpha(q)=1$, as $q \rightarrow \infty$, yields asymptotic optimality.

Let $P(m, k, s)_{q}$ denote the incidence matrix of $\mathcal{C}(m, k, s)_{q}$. We summarize our findings in

Theorem 2 Given integers $1 \leq s<k \leq \frac{1}{2} m$ and a prime power $q$, we have

(i) $P(m, k, s)_{q}$ is a d-disjunct $t \times n$ matrix where $t=q^{m}-1, n=q^{(s+1)(m-k)}, d=q^{k-s}$.

(ii) For any $d \leq q^{k-s}$, the matrix $P(m, k, s)_{q}$ is $d^{z}$-disjunct with $z=q^{k}-1-d\left(q^{s}-1\right)$.

Finally, we explain how good our construction is. Let $t(d, n)$ denote the minimum number of rows for a $d$-disjunct matrix with $n$ columns. In the literature known are the bounds asymptotic in $n$

$$
\Omega\left(1 / d^{2}\right) \leq \frac{\log n}{t(d, n)} \leq O\left((\log d) / d^{2}\right)
$$

( $\log$ is always of base 2). The lower bound is proved in [14], [11], [7] (see also [12], [9, ch.2]) using probabilistic methods. The upper bound is due to D'yachkov and Rykov [11].

Next we compare our construction with the construction in Ngo and Du [18], described in Section 2 (both constructions we take over $G F(q)$ ). In their construction we have $n \leq$ $\alpha(q) q^{(m-k) k}, t \geq q^{(m-r) r}$ (Lemma 2), $d=\frac{q\left(q^{r}-1\right)}{q-1}$ (Theorem 2), and rate $(\log n) / t$.

For the parameters in our construction we use the notation $n_{0}, k_{0}, t_{0}, d_{0}$. Thus, $n_{0}=$ $q^{(s+1)\left(m_{0}-k_{0}\right)}, t_{0}=q^{m_{0}}-1, d_{0} \geq q^{k_{0}-s}$. We put $m_{0}=m, k_{0}=k, s=k-r-1$. Then we have $n_{0}=q^{(k-r)(m-k)}, t_{0}=q^{m}-1, d_{0} \geq q^{r+1}>d$, and rate $\left(\log n_{0}\right) / t_{0}$. A simple calculation shows that $\left(\log n_{0}\right) / t_{0}$ exceeds $(\log n) / t$ by a factor $q^{m(r-1)-r^{2}} \cdot \frac{k-r}{k+1}$.

Let us take now in our construction $q=2, m=2 k$. Then we have $d=2^{k-s}, t=2^{2 k}-1, n=$ $2^{(s+1) k}$ and hence

$$
\frac{\log n}{t}>\frac{(s+1) k}{2^{2 k}}>\frac{s+1}{2^{2 s}} \cdot \frac{\log d}{d^{2}} .
$$

Corollary 1 Given integer $s \geq 1$, our construction gives a class of $d$-disjunct $t \times n$ matrices with parameters $d=2^{k-s}, t=2^{2 k}, n=2^{(s+1) k}$ attaining the upper bound in (3.4), that is rate $(\log n) / t=\Omega\left((\log d) / d^{2}\right)$.

\section{References}

[1] R. Ahlswede, H. Aydinian, and L.H. Khachatrian, On perfect codes and related concepts, Des. Codes Cryptogr. 22, no. 3, 221-237, 2001.

[2] D.J. Balding and D.C. Torney, Optimal pooling designs with error detection, J. Combin. Theory Ser. A 74, no. 1, 131-140, 1996.

[3] R.C. Bose and R.C. Burton, A characterization of flat spaces in a finite geometry and the uniqueness of the Hamming and the MacDonald codes, J. Combin. Theory 1, 96-104, 1996.

[4] A.E. Brouwer, A.M. Cohen, and A. Neumaier, Distance-Regular graphs, SpringerVerlag, Berlin Heidelberg, 1989. 
[5] T. Bu, Partitions of a vector space, Discrete Math. 31, no. 1, 179-83, 1980.

[6] W.E. Clark and M.E.H. Ismail, Binomial and $Q$-binomial coefficient inequalities related to the hamiltonocity of the Kneser graphs and their $Q$-analogues, J. Combin. Theory Ser. A 76, no. 1, 83-98, 1996.

[7] P. Erdös, P. Frankl, and Z. Füredi, Families of finite sets in which no set is covered by the union of $r$ others, Isr. J. Math. 51, no. 1-2, 79-89, 1985.

[8] D.-Z. Du and F.K. Hwang, Combinatorial Group Testing and its Applications, World Scientific, 2nd edit., Singapore, 2000.

[9] D.-Z. Du and F.K. Hwang, Pooling Designs and Nonadaptive Group Testing-Important Tools for DNA sequencing, World Scientific, 2006.

[10] A. D'yachkov, F.K. Hwang, A. Macula, P. Vilenkin, and C. Weng, A construction of pooling designs with some happy surprises, J. Comput. Biology 12, 1129-1136, 2005.

[11] A. D'yachkov, V.V. Rykov, Bounds on the length of disjunctive codes, Problems Inform. Transmission 18, no. 3, 7-13, 1982.

[12] A. D'yachkov, V.V. Rykov, and A.M. Rashad, Superimposed distance codes, Problems Control Inform. 18, no. 4, 237-250, 1989.

[13] E.M. Gabidulin, Theory of codes with maximum rank distance, Problems Inform. Transmission 21, no. 1, 1-12, 1985.

[14] W.H. Kautz and R.C. Singleton, Nonrandom binary superimposed codes, IEEE Trans. Info. Theory 10, 363-377, 1964.

[15] R. Koetter and F.R. Kschischang, Coding for errors and erasures in random network coding, IEEE Trans. Info. Theory 54, no. 8, 3579 - 3591, 2008.

[16] A.J. Macula, A simple construction of $d$-disjunct matrices with certain constant weights, Discrete Math. 162, 311-312, 1996.

[17] A.J. Macula, Error-correcting nonadaptive group testing with $d^{z}$-disjunct matrices , Discrete Appl. Math. 80, 217-222, 1996.

[18] H.Q. Ngo and D.-Z. Du, New constructions of non-adaptive and error-tolerance pooling designs, Discrete Math. 243, 161-170, 2002.

[19] M. Schwartz and T. Etzion, Codes and anticodes in the Grassman graph, J. Combin. Theory Ser. A 97, no. 1, 27-42, 2002.

[20] H. Wang, C. Xing, and R. Safavi-Naini, Linear authentication codes: bounds and constructions, IEEE Trans. Info. Theory 49, no. 4, 866-872, 2003. 